\title{
Indicadores de qualidade no gerenciamento de recursos humanos em enfermagem: elementos constitutivos segundo percepção de enfermeiros*
}

\author{
Quality indicators of the management of human resources in nursing: point of view of \\ registered nurses
}

Indicadores de calidad en la administración de recursos humanos en enfermería: elementos constitutivos según percepción de los enfermeros

\author{
Ana Paula Mirarchi Vieira ${ }^{1}$, Paulina Kurcgant ${ }^{2}$
}

\begin{abstract}
RESUMO
Objetivo: Analisar os significados que os enfermeiros atribuem aos indicadores de qualidade de gerenciamento de recursos humanos e resgatar os elementos de essencialidade constitutivos do processo de gerenciamento de recursos humanos para a construção de indicadores de avaliação em saúde. Método: Foram entrevistados dois enfermeiros do serviço de educação continuada. Resultados: As entrevistas foram analisadas segundo a análise temática e os excertos foram agrupados em três categorias: dimensionamento de pessoal, que alberga as unidades de significado quantitativo de pessoal, qualitativo de pessoal e capacitação de pessoal; a categoria treinamento e desenvolvimento composta pelas unidades de significado número de horas de treinamento de pessoal, Investimento financeiro no treinamento de pessoal e existência de um cronograma de treinamento; e a categoria desempenho pessoal/profissional constituída pelas unidades de significado absenteísmo, número de licenças médicas, rotatividade de pessoal e satisfação/insatisfação no trabalho. Conclusão: As unidades de significado resgatadas e suas respectivas categorias forneceram subsídios significativos para a construção de indicadores de qualidade no gerenciamento de recursos humanos em enfermagem.
\end{abstract}

Descritores: Administração de recursos humanos; Recursos humanos de enfermagem no hospital; Indicadores de serviço.

\begin{abstract}
Objectives: To examine point of view of registered nurses regarding quality indicators of the management of human resources, and to identify essential elements of the management process of human resources for the development of quality indicator in health services. Methods: Data were collected through interviews with two registered nurses from a continuing education department. Results: Three themes emerged from content analysis: (a) scheduling of personnel considering number of staff needed, professional qualification and competency of staff; (b) training and professional development consisting of hours needed for training, financial investment in training, and training schedule; and (c) personal and professional performance concerning absenteism, medical leave, turn-over, and job satisfaction. Conclusion: The idenfication of essential elements of the management process and its sub-categories provide significative support for the development of quality indicators of the management of human resources in nursing.
\end{abstract}

Key Words: Management of Human Resources; Human resources in Nursing; Quality Indicators.

\section{RESUMEN}

Objetivo: Analizar los significados que los enfermeros atribuyen a los indicadores de calidad de la administración de recursos humanos y rescatar los elementos esenciales constitutivos del proceso de administración de recursos humanos para la construcción de indicadores de evaluación de la salud. Método: Fueron entrevistados dos enfermeros del servicio de educación continuada. Resultados: Las entrevistas fueron analizadas según el análisis temático y los fragmentos fueron agrupados en tres categorías: 1) dimensión del personal, que contiene las unidades de significado: cuantitativo de personal, cualitativo de personal y capacitación de personal; 2) la categoría entrenamiento y desarrollo compuesta por las unidades de significado: número de horas de entrenamiento de personal, inversión financiera en el entrenamiento de personal, y existencia de un cronograma de entrenamiento; y 3) la categoría desempeño personal/profesional constituida por las unidades de significado: ausentismo, número de licencias médicas, rotación de personal y satisfacción/insatisfacción en el trabajo. Conclusión: Las unidades de significado rescatadas y sus respectivas categorías entregaron subsidios significativos para la construcción de indicadores de calidad en la administración de recursos humanos en enfermería.

Palabras clave: Administración de recursos humanos; Recursos humanos de enfermería en el hospital; Indicadores de servicio.

\footnotetext{
*Trabalho realizado no Hospital Universitário da Universidade de São Paulo - USP - São Paulo (SP), Brasil.

1 Enfermeira. Bacharel em Enfermagem pela Escola de Enfermagem da Universidade de São Paulo - USP - São Paulo (SP), Brasil.

2 Professora Titular do Departamento de Orientação Profissional da Escola de Enfermagem da Universidade de São Paulo - USP - São Paulo (SP), Brasil.
} 


\section{INTRODUÇÃO}

O presente estudo faz parte do projeto de pesquisa "Indicadores de Qualidade para Avaliação de Serviços de Saúde: Construção de indicadores de qualidade para avaliação de serviços de enfermagem", desenvolvido na Região Sudeste e cujo objetivo é avaliar as ações gerenciais em saúde, segundo indicadores construídos a partir da captação da realidade do processo gerencial em enfermagem.

Para alguns autores ${ }^{(1)}$, a palavra indicador remete a algo que está sendo evidenciado, revelado, demonstrado, tornado patente. $\mathrm{O}$ indicador chama a atenção para determinado aspecto do produto ou de serviço.

Indicador de qualidade, por sua vez, é uma medida quantitativa de uma determinada característica associada à qualidade julgada pelo cliente ${ }^{(2)}$. A finalidade dos indicadores é analisar as condições do processo e do produto/ serviço e compará-las com os padrões estabelecidos, contribuindo para a verificação de desvios e conseqüente busca de melhorias, mantendo e aprimorando o nível de qualidade $^{(1-2)}$. A realidade abordada pelos indicadores deve ser relevante àquelas pessoas que lidam com a tomada de decisão, pois serão essas as líderes do processo de mudança ${ }^{(3)}$.

A relação entre indicadores e serviços de saúde é evidenciada nas definições: indicadores são medidas usadas para ajudar a descrever uma situação existente, avaliar mudanças ou tendências durante um período de tempo e avaliar, em termos de qualidade e quantidade, as ações de saúde executadas.

Um indicador não mede diretamente a qualidade de um serviço, mas sua análise permite uma comparação entre um fato real e a meta que se quer atingir, ou até possibilitar a criação de parâmetros, sejam eles internos ou externos, em uma organização de saúde. Portanto, um indicador pode ser compreendido como um dado que gera informações sobre assuntos que merecem uma atenção e que possibilita uma revisão de situações específicas ${ }^{(1)}$.

O Sistema de Indicadores Padronizados para Gestão Hospitalar (SIPAGEH) e o Programa de Estudos Avançados em Administração Hospitalar e de Sistemas de Saúde adotam, entre outros, como indicadores de qualidade hospitalar: Rotatividade, Absenteísmo, Tempo Médio de Permanência de pacientes, Taxa de Infecção Hospitalar e Taxa de Ocupação Hospitalar ${ }^{(4-5)}$.

No que tange ao serviço de enfermagem, indicadores relacionados à assistência são amplamente utilizados. A American Nursing Association (ANA) sugere como indicadores para a avaliação da qualidade da assistência de Enfermagem a Taxa de Infecção Hospitalar (para Infecção do Trato Urinário e Cateter Venoso Central); a Taxa de Acidentes com o Paciente (como quedas que levam a ferimentos); a Satisfação do Paciente com os Cuidados de Enfermagem; Satisfação do Paciente com o Controle da Dor; a Satisfação do Paciente sobre a Informação Educacional Recebida; Manutenção da Integridade da Pele (em relação a úlceras por pressão), Satisfação da Equipe de Enfermagem, Número Total de Enfermeiros/Técnicos e Auxiliares de
Enfermagem e Taxa de Horas de Enfermagem por Pacientes/ $\operatorname{Dia}^{(6)}$.

Entretanto, no âmbito da gerência e, mais especificamente, no que diz respeito à gestão de recursos humanos em saúde e em enfermagem, o uso de indicadores não tem sido adotado e chama a atenção o seu reduzido número.

A American Society for Training e Development tem utilizado apenas dois indicadores referentes à avaliação do gerenciamento de pessoal ${ }^{(7)}$ :

- percentual de investimento em treinamento por ano por funcionário e,

- número de horas de treinamento por ano por funcionário

Para melhor compreensão de uma situação estudada, o uso de apenas um indicador não é suficiente e a elaboração, associação e até mesmo a comparação entre vários indicadores permitem uma análise mais fidedigna da realidade ${ }^{(3)}$.

Devido à complexidade e à diversidade dos fatores que influenciam o gerenciamento do trabalho de enfermagem, apenas horas de treinamento não avaliam essa função administrativa sob a responsabilidade do enfermeiro. Assim, o número reduzido de ferramentas que vem sendo adotadas para a avaliação da qualidade do gerenciamento de pessoal em saúde, justifica a urgência que se faz presente na construção de indicadores de qualidade para a avaliação de gerenciamento de recursos humanos em enfermagem.

Nesse contexto, o presente estudo teve como objetivos:

- Analisar os significados que os enfermeiros atribuem aos indicadores de qualidade de gerenciamento de recursos humanos;

- Resgatar, ouvidos os enfermeiros, os elementos de essencialidade constitutivos do processo de gerenciamento de recursos humanos para a construção de indicadores de avaliação em saúde.

\section{METODOLOGIA}

O estudo é do tipo descritivo-exploratório e, como estudo exploratório, possibilita o necessário contato com o objeto a ser desvendado que, no caso, são os indicadores de qualidade no gerenciamento de recursos humanos. O caráter descritivo se mostra através dos objetivos que são definidos e dirigidos para a elucidação do objeto pesquisado e resgatado segundo a percepção de enfermeiros do Setor da Educação Continuada.

Para tanto, foi adotada a metodologia da pesquisa qualitativa, uma vez que cada sujeito pode contribuir, com sua perspectiva do problema pesquisado, permitindo ao pesquisador resgatar informações sobre o problema, a partir da ótica de quem vivencia a situação analisada.

O locus do presente estudo foi o Hospital Universitário da Universidade de São Paulo (HU-USP), e os sujeitos do estudo foram dois enfermeiros que participaram, na sua prática cotidiana em diferentes momentos, do gerenciamento de recursos humanos no HU-USP, por trabalharem ou terem trabalhado no Serviço de Apoio à Educação desta instituição.

Os dados foram coletados através da técnica da entrevista semi-estruturada e orientada por duas questões: O que você 
entende por indicador de qualidade de gerenciamento de recursos humanos em enfermagem? $\mathrm{Na}$ sua vivência, o que você levaria em consideração para avaliar a qualidade no gerenciamento de recursos humanos em enfermagem?

Após serem gravadas, as entrevistas foram transcritas e analisadas gerando as categorias empíricas apreendidas nas entrevistas e revelando os elementos constitutivos de indicadores de gestão de pessoal.

Optou-se pela análise de conteúdo, definida como "um conjunto de técnicas de análise de comunicação visando obter, por procedimentos sistemáticos e objetivos de descrição do conteúdo das mensagens, indicadores (quantitativos ou não) que permitam a inferência de conhecimentos relativos às condições de produção/recepção destas mensagens"(8). Para a análise de conteúdo, adotou-se a técnica da análise temática que "consiste em descobrir os núcleos de sentido que compõem uma comunicação cuja presença ou freqüência signifiquem alguma coisa para o objetivo analítico visado"(8).

Para a operacionalização da pesquisa foram considerados os seguintes procedimentos:

- Aprovação do Projeto pelo Comitê de Ética e Pesquisa do HU-USP;

- Solicitação de autorização da instituição locus da pesquisa mediante apresentação do projeto de pesquisa e do parecer do Comitê de Ética;

- Solicitação para entrevista com os enfermeiros que voluntariamente se dispuseram a participar da pesquisa, após conhecimento, aceitação e assinatura do Termo de Consentimento Livre e Esclarecido.

- Denominação E1 e E2 para os enfermeiros colaboradores da pesquisa.

\section{RESULTADOS}

Através da análise temática das entrevistas foram apreendidas três categorias descritas a seguir.

\section{Dimensionamento de pessoal}

As unidades de significado destacadas foram: quantitativo de pessoal, qualitativo de pessoal e capacitação de pessoal.

Os sujeitos do estudo demonstraram preocupação em relação à quantidade de enfermeiros, auxiliares e técnicos de enfermagem disponíveis para atender a demanda do setor, e a formação ou qualificação dos mesmos para desempenharem suas respectivas atividades.

"Para este dado hospital, ou para esta unidade on para este setor, eu tenho que ter profissionais em quantidade adequada, de acordo com a necessidade de cuidados que eu tenho aqui. Então, tem que ter número adequado por leito, por número, por complexidade(...) eu penso além da quantidade, eu penso assim na qualidade que tem esse profissional, ou seja, qual é a formação que ele tem para estar exercendo a função no local que ele está trabalhando". (E1)

O preparo do enfermeiro enquanto líder da equipe de enfermagem também foi destacado por E1:
“(...) o preparo que as pessoas que ocupam cargo de liderança possuem para exercer a função. Então, se é uma enfermeira que é chefe, qual o preparo que ela tem, porque a gente tem que ter é - preparo adequado para dar conta das demandas que aquela função vai exigir ou não."

\section{Treinamento e desenvolvimento}

As unidades de significado depreendidas foram: número de horas de treinamento de pessoal, investimento financeiro no treinamento de pessoal e existência de um cronograma de treinamento.

Esta categoria abrange temas que aprofundam a questão da formação profissional citada na categoria anterior. Os entrevistados destacam a postura da instituição de saúde em relação ao desenvolvimento dos seus colaboradores, no que tange ao tempo e recurso financeiro despendidos para o treinamento desses.

“(...) quanto que existe de investimento neste profissional para que ele dê conta de realizar as suas funções (...) e até mesmo os cursos que a instituição oferece". (E1)

E1 questiona a periodicidade do treinamento, ao dizer que:

"não é um treinamento boje e outro daqui a um ano, é um treinamento periódico, sistemático, ele está sendo intencionalmente controlado".

\section{Desempenho profissional/pessoal}

Absenteísmo, número de licenças médicas (em determinado período de tempo), rotatividade de pessoal e satisfação/insatisfação no trabalho.

Os entrevistados manifestaram fatores relacionados ao desempenho profissional e pessoal que refletem as condições de gerenciamento de recursos humanos da instituição, como a taxa de ausência e a rotatividade dos funcionários:

“(...) uma coisa que en veria como indicador de qualidade é a taxa de ausência dos funcionários, que é o absenteísmo, o quanto eu tenho de falta de profissional" (E1).

"Se en tiver um lugar que eu percebo que as pessoas rodam com muita freqüência, algum problema eu tenho. Então eu vou talvez inferir que um lugar que eu tenho uma baixa taxa de rotatividade, as condições podem ser boas”. (E1)

E1 também coloca a "questão da satisfação do profissional" e o círculo vicioso das licenças médicas. Uma vez que "se eu tiver uma equipe (...) com quantitativo que não é adequado (e) se você tiver uma complexidade maior de atendimentos (...) certamente você vai ter uma taxa maior de ausência de pessoal" devido afastamentos por motivos de saúde. Consequentemente, haverá menos funcionários para trabalhar, que, por sua vez, ficarão sobrecarregados e mais suscetíveis aos riscos de doenças ocupacionais. 


\section{DISCUSSÃO}

A definição de dimensionamento de pessoal, segundo alguns autores ${ }^{(9)}$, é a "etapa inicial do processo de provimento de pessoal, que tem por finalidade a previsão da quantidade de funcionários por categoria, requerida para suprir as necessidades de assistência de enfermagem, direta e indiretamente prestada à clientela".

A Resolução n 293/2004 do COFEN estabelece que o dimensionamento do pessoal de enfermagem deve basear-se nas características relacionadas à empresa (políticas institucionais, porte, tipo de serviço prestado), ao serviço de enfermagem (modelo assistencial) e à clientela (sistema de classificação de pacientes). A Resolução também coloca que o dimensionamento de pessoal deve acrescer uma porcentagem de funcionários para cobrir as ausências previstas e não previstas ${ }^{(10)}$.

Estabelecer, portanto, um adequado dimensionamento do quadro de enfermagem é fundamental para se garantir uma assistência de qualidade, pois estabelece o quantitativo de pessoal necessário para atender a demanda assistencial da clientela atendida.

Em relação ao preparo do enfermeiro enquanto líder da equipe, na literatura são consideradas características positivas num líder a credibilidade, a comunicabilidade, o conhecimento, o bom relacionamento, o envolvimento e a segurança. Em contrapartida, são considerados pontos negativos a insegurança e a comunicação ineficaz ${ }^{(11)}$.

Para alguns autores, treinamento "constitui ação sistematizada de capacitação e adaptação do indivíduo em uma situação profissional específica, realizada em curto prazo", enquanto desenvolvimento "propõe ações voltadas para o aperfeiçoamento e o crescimento pessoal e profissional do indivíduo, realizado em médio e longo prazos" ${ }^{\text {(12). }}$.

Em relação ao número de horas de treinamento de pessoal, o SIPAGEH e a ASTD já utilizam este indicador para avaliar a qualidade do serviço prestado ${ }^{(4,7)}$.

No que tange ao investimento financeiro despendido no treinamento de pessoal, estudopioneiro nesta temática, mostra os custos diretos envolvidos no processo de treinamento em Reanimação Cardio-Respiratória para técnicos e auxiliares de Enfermagem na Unidade de Terapia Intensiva (UTI) e Unidade de Terapia Semi-Intensiva de um Hospital Escola em São Paulo ${ }^{(13)}$.

Sobre a periodicidade de treinamento, os estudos são ainda escassos na área da saúde. Autores ${ }^{(14)}$, ao analisarem a temática da Educação Continuada nas UTIs dos hospitais do Município de São Paulo, abordaram a questão da periodicidade dos treinamentos através da existência de programas de reciclagem e atualização.

Quanto ao absenteísmo, as ausências previstas referemse às folgas semanais, feriados e férias, enquanto as ausências não previstas englobam faltas, licenças e suspensões ${ }^{(9)}$.

A qualidade e a quantidade da assistência direcionada ao cliente são prejudicadas devido a falhas na organização do trabalho como conseqüência das ausências dos profissionais.
Esta situação também afeta os enfermeiros com cargos de chefia, dado que são estes os responsáveis pela resolutividade deste tipo de problema. Além disso, as causas do absenteísmo estão não apenas na pessoa, mas também na empresa ${ }^{(15)}$.

Ainda de acordo com o estudo realizado ${ }^{(15)}$, de um total de 681 ausências não previstas ocorridas em um ano, 72,6\% foram por motivo de doença, ocasionando 1491 dias perdidos de trabalho. Logo, é nítido o impacto do número de licenças médicas na qualidade do serviço prestado nas instituições de saúde.

Assim como o absenteísmo, a rotatividade de pessoal também prejudica a qualidade da assistência, uma vez que os funcionários que permanecem no serviço ficam sobrecarregados, gerando descontentamento e insatisfação, além de onerar economicamente a empresa, pois esta gasta mais ao admitir um novo colaborador ${ }^{(16)}$.

Em relação à satisfação/insatisfação no trabalho, autores ${ }^{(17)}$ reúnem os resultados obtidos a partir de pesquisas sobre satisfação no trabalho do enfermeiro. Como fatores de satisfação, encontram o reconhecimento, a responsabilidade, autonomia e possibilidade de oferecimento de uma assistência integral ao paciente. Quanto aos fatores de insatisfação, foram indicados: nível salarial, qualidade da supervisão, relacionamento com a equipe de trabalho e condições de trabalho ${ }^{(17)}$. Entretanto, estes dois últimos itens surgem como fatores motivacionais para o trabalho em uma outra pesquisa ${ }^{(18)}$, refletindo, dessa forma, a necessidade de maiores pesquisas nesse campo.

\section{CONSIDERAÇÕES FINAIS}

A análise das falas permitiu identificar como enfermeiros de um Serviço de Educação Continuada de um Hospital Universitário percebem o significado de indicadores de qualidade na avaliação dos serviços de saúde.

Assim, permitiu resgatar que os enfermeiros que vivenciam as atividades de educação permanente consideram o dimensionamento, o treinamento o desenvolvimento e o desempenho profissional/pessoal como elementos constitutivos de indicadores de qualidade.

$\mathrm{O}$ número de entrevistados poderia ser considerado um viés desta pesquisa, porém, tratando-se de uma pesquisa qualitativa e da larga experiência dos dois colaboradores como gerentes do Serviço de Apoio Educacional do HUUSP, o conteúdo apreendido permitiu atender aos objetivos propostos.

A discussão também foi dificultada pela escassez de literatura sobre indicadores de gerenciamento de recursos humanos, para comparar os resultados obtidos, evidenciando o ineditismo do presente estudo.

Por fim, este estudo vai ao encontro do que é preconizada hoje no gerenciamento de recursos humanos, o desenvolvimento e o bem-estar dos agentes, considerando que as empresas vêm investindo, cada vez mais, em políticas que atendam a estes preceitos. 


\section{REFERÊNCIAS}

1. Bohomol E, D’Innocenzo M, Cunha ICKO. Indicadores de qualidade - conceitos e sistemas de monitoramento. Cad Centro Universitário S Camilo. 2005;11(2):75-81.

2. Mello JB, Camargo MO. Qualidade na saúde: práticas e conceitos. Normas ISO nas áreas médico-hospitalar e laboratorial. São Paulo: Best Seller; 1998.

3. Malik AM, Schiesari LMC. Qualidade na gestão local de serviços e ações de saúde. São Paulo: Faculdade de Saúde Pública da Universidade de São Paulo; 1998.

4. SIPAGEH - Sistema de Indicadores Padronizados para Gestão Hospitalar [Internet]. [citado 2006 maio2]. http:/ /www.economicas.unisinos.br/sipageh/indic.php

5. Hospital das Clínicas da Faculdade de Medicina da Universidade de São Paulo. PROAHSA - Programa de Estudos Avançados em Administração Hospitalar e Sistemas de Saúde [Internet]. [citado 2006 Maio 2] Disponível em: http://www.hcnet.usp.br/proahsa/ index.htm.

6. Denser CPAC. Indicadores: instrumento para a prática de enfermagem com qualidade. In: Bork AMT, Minatel VF, organizadoras. Enfermagem de excelência: da visão a ação. Rio de Janeiro: Guanabara Koogan; 2003. v. 1, p. 91-100.

7. Jericó MC, Castilho V. Análise de alguns indicadores relacionados aos recursos humanos de uma organização hospitalar. Nursing (São Paulo). 2004;7(74): 23-7.

8. Minayo MCS. O desafio do conhecimento: pesquisa qualitativa em saúde. 8a. ed. São Paulo: Hucitec; 2004.

9. Gaidzinski RR, Fugulin FMT, Castilho V. Dimensionamento de pessoal de enfermagem em instituições de saúde. In: Kurcgant P, coordenadora. Gerenciamento em enfermagem. Rio de Janeiro: Guanabara Koogan; 2005. p.125-37.

10. Portal COREN-SP. Resolução COFEN n 293/2004.
Fixa e Estabelece Parâmetros para o Dimensionamento do Quadro de Profissionais de Enfermagem nas Unidades Assistenciais das Instituições de Saúde e Assemelhados [Internet]. [citado em 2009 Mar 30]. Disponível em: http://www.coren-sp.gov.br/drupal6/node/3538

11. Simões ANA, Fávero N. O desafio da liderança para o enfermeiro. Rev Latino-am Enfermagem. 2003;11(5): 567-73.

12. Peres HHC, Leite MMJ, Mira VL. Educação continuada: recrutamento e seleção, treinamento e desenvolvimento, e avaliação de desempenho profissional. In: Kurcgant P, coordenadora. Gerenciamento em enfermagem. Rio de Janeiro: Guanabara Koogan; 2005. p.138-56.

13. Follador NN, Castilho V. O custo direto do Programa de Treinamento em ressuscitação cardiopulmonar em um Hospital Universitário. Rev Esc Enferm USP. 2007;41(1): 90-6.

14. Koizumi MS, Kimura M, Miyadahira AMK, Cruz DALM, Padilha KG, Sousa RMC, Altimari PDM. Educação continuada da equipe de enfermagem nas UTIs do município de São Paulo. Rev Latinoam Enferm. 1998;6(3):33-41.

15. Silva DMPP, Marziale MHP. Absenteísmo de trabalhadores de enfermagem em um hospital universitário. Rev Latino-am Enfermagem. 2000;8(5):44-51.

16. Nomuraa FH, Gaidzinski RR. Rotatividade da equipe de enfermagem: estudo em hospital-escola. Rev Latino-am Enfermagem. 2005;13(5):648-53.

17. Del Cura MLA, Rodrigues ARF. Satisfação profissional do enfermeiro. Rev Latino-am Enfermagem. 1999;7(4):21-8.

18. Batista AAV, Vieira MJ, Cardoso NCS, Carvalho GRP. Fatores de motivação e insatisfação no trabalho do enfermeiro. Rev Esc Enferm USP. 2005; 39(1):85-91. 